\begin{tabular}{|c|l|}
\hline Title & A mmonia Channel Couples Glutaminase with Transamidase Reactions in GatCA B \\
\hline Author(s) & Nakamura, A kiyoshi; Y ao, Min; Chimnaronk, Sarin; Sakai, Naoki; Tanaka, Isao \\
\hline Citation & $\begin{array}{l}\text { Science, 312(5782), 1954-1958 } \\
\text { https://doi.org/10.1126/science.1127156 }\end{array}$ \\
\hline Issue Date & 2006-06-30 \\
\hline Doc URL & http://hdl.handle.net/2115/14490 \\
\hline Type & article \\
\hline File Information & nakamura.pdf \\
\hline
\end{tabular}

Instructions for use 


\section{Ammonia Channel Couples Glutaminase with Transamidase Reactions in GatCAB}

One-sentence summary: A series of X-ray crystal structures of bacterial Glu-tRNA ${ }^{\text {Gln }}$-dependent amidotransferase (GatCAB) in the apo form and in the substrate-bound states, combined with the biochemical studies, elucidate the sophisticated mechanism to synthesize Gln-tRNA ${ }^{\text {Gln }}$, coupling the glutaminase with the kinase and transamidase reactions by a characteristic ammonia channel $30 \AA$ in length.

\section{Akiyoshi Nakamura, ${ }^{1}{ }^{*}$ Min Yao, ${ }^{1} *$ Sarin Chimnaronk, ${ }^{1,2}$ Naoki Sakai, ${ }^{1}$ and Isao Tanaka ${ }^{1} \dagger$}

${ }^{1}$ Faculty of Advanced Life Sciences, Hokkaido University, Sapporo 060-0810, Japan

${ }^{2}$ Institute of Molecular Biology and Genetics, Mahidol University, Salaya Campus, Nakornpathom 73170, Thailand

*These authors contributed equally to this work.

$\dagger$ To whom correspondence should be addressed.

E-mail: tanaka@castor.sci.hokudai.ac.jp 


\begin{abstract}
The formation of glutaminyl transfer tRNA (GIn-tRNA $\left.{ }^{\text {Gln }}\right)$ differs among the three domains of life. Most bacteria employ an indirect pathway to produce GIn-tRNA ${ }^{\text {Gln }}$ by a heterotrimeric amidotransferase CAB (GatCAB) that acts on the misacylated Glu-tRNA $^{\text {Gln }}$. Here we describe a series of crystal structures of intact GatCAB from Staphylococcus aureus, in the apo form, and in the complexes with glutamine, asparagine, $\mathrm{Mn}^{2+}$, and adenosine triphosphate analog. Two identified catalytic centers for the glutaminase and transamidase reactions are markedly distant but connected by a hydrophilic ammonia channel $30 \AA \AA$ in length. Further, we show that the first U-A base pair in the acceptor stem and the D loop of tRNA ${ }^{\text {Gln }}$ serve as identity elements essential for discrimination by GatCAB, and propose a complete model for the overall concerted reactions to synthesize GIn-tRNA ${ }^{\text {Gln }}$.
\end{abstract}


Accurate translation of the genetic code into proteins requires high efficiency and fidelity of tRNA-aminoacylation (1). This vital process is governed by a fundamental family of enzymes called aminoacyl-tRNA synthetases (aaRS), which catalyze the direct attachment of amino acids to the 3'-ends of the corresponding tRNAs. However, Gln-tRNA $^{\text {Gln }}$ is synthesized by two different pathways in nature (2-4). In the eukaryotic cytoplasm and in some bacteria, glutamine is ligated directly to tRNA ${ }^{\text {Gln }}$ by glutaminyl-tRNA synthetase (GlnRS), whereas a non-canonical indirect pathway is employed in the majority of bacteria and all archaea, which do not possess GlnRS, through a two-step process $(2,5)$. During the first step, $\mathrm{tRNA}^{\mathrm{Gln}}$ is misacylated with glutamic acid by a nondiscriminating glutamyl-tRNA synthetase (GluRS) which recognizes both tRNA ${ }^{\text {Glu }}$ and tRNA ${ }^{\text {Gln }}$. Thereafter, the mischarged Glu-tRNA ${ }^{\text {Gln }}$ is transformed into Gln-tRNA ${ }^{\text {Gln }}$ by Glu-tRNA ${ }^{\text {Gln }}$-dependent amidotransferase (Glu-AdT). Glu-AdT converts Glu-tRNA ${ }^{\text {Gln }}$ into Gln-tRNA $^{\text {Gln }}$ by initially activating Glu-tRNA ${ }^{\text {Gln }}$ into $\gamma$-phosphoryl-Glu-tRNA ${ }^{\text {Gln }}$ at the expense of ATP, which is subsequently transamidated into Gln-tRNA ${ }^{\text {Gln }}$ using ammonia generated by hydrolysis of glutamine or asparagine. Moreover, Asn-tRNA ${ }^{\text {Asn }}$ can also be synthesized through such an indirect pathway by Asp-tRNA ${ }^{\text {Asn }}$-dependent amidotransferase (Asp-AdT) in organisms lacking asparaginyl-tRNA synthetase $(6,7)$.

Bacterial Glu-AdTs are heterotrimeric proteins composed of A, B, and C subunits, and have therefore been named GatCAB, while archaea employ the heterodimeric GatDE enzymes $(8,9)$. Although bacterial GatCAB acts as both Glu- and Asp-AdT, GatDE specifically recognizes only Glu-tRNA ${ }^{\text {Gln }}(9)$. It is intriguing that the three domains in nature use distinct enzymes to synthesize Gln-tRNA ${ }^{\text {Gln }}$ in protein synthesis. Based on the primary sequences analysis, GatA is homologous to the amidase enzymes, whereas GatD is closer to the L-asparaginases $(9,10)$. Therefore, GatA and GatD are structurally different but are assumed to play the same role as the glutaminase subunit for ammonia production. On the other hand, GatB is highly related exclusively to its archaeal counterpart, GatE (9, 
10). GatE alone can produce $\gamma$-phosphoryl-Glu-tRNA ${ }^{\text {Gln }}$, which suggests that the activation of Glu-tRNA $^{\text {Gln }}$ is achieved only by the GatB or GatE subunits (11). Notably, the glutaminase activity and the activation of Glu-tRNA ${ }^{\text {Gln }}$ into $\gamma$-phosphoryl-Glu-tRNA ${ }^{\text {Gln }}$ are tightly coupled upon the binding of Glu-tRNA ${ }^{\text {Gln }}(11,12)$. Hence, these modules of distinct functional enzymes were assembled to form a more sophisticated protein complex, and acquired novel mechanisms to expand the genetic code during evolution.

Recently, the $\alpha_{2} \beta_{2}$ tetramer structure of archaeal GatDE from Pyrococcus abyssi was reported at 3.0 A resolution (10); however, the precise mechanisms for each of the overall reactions coupling the glutaminase with the kinase and transamidase activities, regulated by Glu-tRNA $^{\text {Gln }}$, remain elusive. To address this issue, we initiated the crystal structure analysis of bacterial GatCAB from the pathogenic bacterium, S. aureus Mu50 strain. The crystal structure of apo GatCAB was solved at a resolution of $2.5 \AA$, by the single wavelength anomalous diffraction (SAD) method, and refined to a final $R$-factor of $23.8 \%$ $(R$-free $=27.5 \%)$ using the LAFIRE program running with CNS (13-15) (Fig. 1A). The asymmetric unit contained one GatCAB heterotrimeric molecule, in which the flexible C-terminal region of GatB (residues 412 to 475) was disordered and could not be modeled. The structure immediately highlights an important role of the $\mathrm{C}$ subunit as a stabilizer of the protein complex. GatC is featured by an unstructured extended loop flanked by two helices at its $\mathrm{N}$-terminus and two $\beta$-strands at its $\mathrm{C}$-terminus (Fig. 1B). GatC wraps around the interface region as a belt and makes extensive interactions with both GatA and GatB. The amphipathic $\mathrm{C} \alpha 1$ and $\mathrm{C} \alpha 2$ helices form a helical bundle with the hydrophobic core of GatA (A $\alpha 9-11,13)$, thus relieving the local hydrophobicity (Fig. 1C). The internal loop region of GatC crosses over the loop-rich side of GatB, stabilized through a hydrogen bond network by two invariant Arg64 and Asp66 residues of GatC (Fig. 1D). The association of GatAB complex is further assured by the hydrophobic interactions of $\mathrm{C} \alpha 3$ helix of GatC with GatAB. Interestingly, the C-terminal $\mathrm{C} \beta 1-2$ strands make an antiparallel sheet with a 
$\beta$-hairpin (B $\beta 8$-9) of GatB, forming a four-stranded sheet, resembling the corresponding region in GatDE (Fig. 1E).

GatA is a single-domain protein consisting of a central, mixed 11-stranded $\beta$-sheet core covered by double layers of $\alpha$-helices on the top and bottom (fig. S2). The highest structural similarity was found in malonamidase E2 (MAE2) from Bradyrhizobium japonicum [Protein Data Bank (PDB) code 1OCK (16))], with a root mean square deviation of $2.1 \AA$ for 405 pairs of $\mathrm{C} \alpha$ atoms compared. GatA faces its loop-rich plane to and caps a loop-rich side of the cradle domain of GatB, burying $7 \%\left(2680 \AA^{2}\right)$ of the total surface area in the inter-subunit interface (Fig. 1A). There is a putative ammonia channel (described below) running through the middle of the interface, which is surrounded by two layers of interfacial interactions (fig. S1). The inner layer encircling the channel is composed of polar residues, whereas the outer layer is mostly hydrophobic; therefore, GatA is moderately attached to GatB through both hydrogen-bonding and hydrophobic interactions.

Seeking the molecular mechanism of the glutaminase reaction in GatA, we soaked GatCAB crystals in $1 \mathrm{mM}$ glutamine and determined the cocrystal structure by molecular replacement. The glutamine was found in the center of GatA constructed by the amidase signature sequence (fig. S2). The amide group of glutamine is recognized by Asp425, and the carboxyl group is recognized by Arg358 (Fig. 2A). The side chain of the glutamine is located close to the conserved Ser-cis-Ser-Lys catalytic scissors: Ser178, Ser154 and Lys79. Consistent with MAE2 (16), in GatA, Ser154 is located in the loop enriched in small residues (glycine, serine, and alanine) and forms an unusual cis conformation, which enables itself to deprotonate Ser178. Surprisingly, Ser178 made a tight covalent bond (1.44 $\AA$ ) with the amide carbonyl carbon atom of the glutaminyl side chain, revealing the tetrahedral covalent intermediate, which is stabilized by the oxyanion hole constructed by the backbone nitrogen atoms of Thr175, Gly176, Gly177, and Ser178 (Fig. 2A). We further observed a continuous electron density sprouting out from the amide group of the side 
chain of glutamine, which may correspond to amide ammonia liberated from the substrate. On the opposite site is a water molecule, which was not observed in the apo GatCAB crystal, anchored by hydrogen bonds with Ser153, Ser178, and the amide group of the substrate itself, which is in the vicinity of hydrolysis of the enzyme-acyl intermediate. These observations clearly indicated that GatA employs the same hydrolysis mechanism as the amide hydrolysis activity of amidase. Curiously, in contrast to the previous study on GatDE from Pyrococcus abyssi that showed glutaminase activity only in the presence of Glu-tRNA $^{\text {Gln }}(10,11)$, GatCAB intrinsically possesses basal glutaminase activity without binding of substrate tRNA [in the current structure and by kinetics studies (12)], which suggests that the conformational rearrangement of the glutaminase active site of GatA is not involved in regulation of the concerted reactions.

GatB is organized into two domains connected by a characteristic $\sim 60 \AA$ elongated linker loop (fig. S4). The globular "cradle" domain is topologically unique, and can be distinguished from GatE by two insertions on each side of the cradle (10). GatE has an insertion domain, resembling those found in bacterial AspRS, between $\mathrm{B} \alpha 3$ and $\mathrm{B} \beta 20$, and an antiparallel $\beta$-sheet inserted between $\mathrm{B} \beta 1$ and $\mathrm{B} \alpha 1$ (fig. S3). The auxiliary $\mathrm{C}$-terminal helical domain is built by an $\alpha$-helical bundle architecture with seven traceable helices. The disordered C-terminal region of GatB (residues 412 to 475 ) is presumed to form another three-helix bundle as indicated by the sequence identity to the C-terminus of uncharacterized Yqey protein (PDB code:1NG6). An obvious density peak of $\mathrm{Mg}^{2+}$ ion was observed at the bottom of the cradle domain coordinated to three conserved residues consisting of His12, Glu124, and Glu150, and to three water molecules (Fig. 2B). To underline the molecular basis for the mechanism of the kinase activity, we co-crystallized GatCAB with the $\mathrm{ADP}-\mathrm{AlF}_{4}{ }^{-}$compound as an ATP analog. The $3.35 \AA 2 F o-F C$ and $F o-F C$ maps clearly indicated that ADP sits on strands $B \beta 1$ and $B \beta 11$ at the bottom of the cradle domain, with a bound water molecule (Fig. 2B). The adenosine base dips into a 
hydrophobic pocket, created by Val6, Phe205, and Pro155. N1 and N6 are strictly recognized by an invariant Ser196 residue in agreement with the previous report that GTP could not serve as a phosphate donor for GatCAB (17). The hydroxyl groups of ribose interact with the backbone of a loop between $\mathrm{B} \alpha 2$ and $\mathrm{B} \beta 10$, whereas the ribose $\mathrm{O} 4$ is recognized by the conserved Asn194 residue. The $\beta$-phosphate is at an appropriate distance for hydrogen bonding with the conserved Glu10 and an anchored wat95 molecule.

Cautious inspection of the $F o-F C$ difference electron density map from a crystal derivatized with $\mathrm{MnCl}_{2}$ revealed a pair of $\mathrm{Mn}$ cations in the active site of GatB (fig. S4). The prominent $\mathrm{Mn}^{2+}$-binding site is identical to the $\mathrm{Mg}^{2+}$ site in the native crystal. The second transient binding site is located adjacent to the first (a distance of $6.3 \AA$ ), coordinated to the conserved acidic residue cluster formed by Glu10, Asp192, and Glu210. This position corresponds to the wat95 water molecule in the $\mathrm{ADP}-\mathrm{AlF}_{4}{ }^{-}$structure, and is at an appropriate distance to make interactions with $\beta$ - and $\gamma$-phosphates of ATP. These results strongly suggested that GatB (and plausibly GatE) uses a two-metal-ion mechanism of catalysis, which is reminiscent of the mechanism employed by glutamine synthetases in amino acid biosynthesis (18-21). We propose that the primary $\mathrm{Mg}^{2+}$-binding site should be responsible for coordinate bonding, recognition, and positioning of the $\gamma$-carboxyl group of the glutamate attached to the 3 '-end of tRNA ${ }^{\text {Gln }}$ (fig. S6). On the other hand, the transient $\mathrm{Mg}^{2+}$-binding site is likely to interact with $\beta$ and $\gamma$ phosphates of ATP and to participate in phosphoryl transfer by polarizing the $\gamma$-phosphate group of ATP, promoting the nucleophilic attack to form a $\gamma$-phosphoryl-Glu-tRNA ${ }^{\text {Gln }}$ (fig. S6).

The apo GatCAB structure revealed a distance of $30 \AA$ that separates the catalytic sites at which the glutaminase reaction takes place, and at which the CCA-end of tRNA binds. We found that two reaction centers in GatA and GatB are connected by a penetrable tunnel lined with a continuous succession of conserved polar residues (Fig. 2, C and D). In our GatCAB/glutamine complex structure, this tunnel is thoroughly hydrophilic and is 
filled with a row of solvent molecules indicating the actual route of ammonia traveling from the amino acid pocket in GatA to reach the $\gamma$-phosphoryl-Glu-tRNA ${ }^{\text {Gln }}$ in GatB. Strictly conserved positive and negative residues, designating the ammonia channel, are located alternately along the course (Fig. 2, C and D). This may imply a "proton-relay mechanism" by which ammonia is dispersed from one site to the other by repeating protonation and deprotonation with these successive conserved polar residues. This hypothesis is reconciled by the positioning of Thr175 A at the entrance of the channel and Lys79 $\mathrm{B}$ at the exit as the proton donor and acceptor, respectively. However, additional biochemical and structural studies should be required to clarify the precise mechanism of ammonia transport in GatCAB. In addition, distinct ammonia tunnels have been described in multistep enzymatic proteins that hydrolyze glutamine to obtain ammonia as a substrate for a second reaction, such as asparagine synthetase $\mathrm{B}$, imidazole glycerol phosphate synthase, or glutamine phosphoribosylpyrophosphate, as well as in the case of the transmembranal ammonia transporter proteins (22-25). All of these adopt a dissimilar architecture of hydrophobic tunnel ( $\sim 20 \AA$ in length) suggesting that ammonia $\left(\mathrm{NH}_{3}\right)$, but not ammonium ion $\left(\mathrm{NH}_{4}{ }^{+}\right)$, is transported. Therefore, GatB is idiosyncratic not only in its folding but also in its mechanism of the intramolecular ammonia transportation. According to the program CAVER (26) and an in-house program used in calculation of the tunnel of GatCAB, we readily noticed a bottleneck in the middle of the path of ammonia travel (Fig. 2C). The channel is constricted by a completely conserved Glu125 residue residing in $\mathrm{B} \beta 7$ of GatB, which makes a salt bridge with the invariant Lys88 on a loop between $B \beta 4$ and $B \beta 3$. As the efficiency of glutamine hydrolysis is known to be stringently dependent on binding of Glu-tRNA ${ }^{\text {Gln }}$, but neither uncharged tRNA nor Gln-tRNA ${ }^{\text {Gln }}$ (12), the Glu-tRNA ${ }^{\text {Gln }}$ recognition must induce as yet uncharacterized conformational changes. We postulate that there is an open-close motion of Glu125 as a gate susceptible to tRNA binding. Juxtaposed to Glu125 is a conserved Asp126 residue, which forms a tight salt 
bridge with the invariant $\operatorname{Arg} 190$ on the opposite $\mathrm{B} \beta 11$ strand, resulting in a twist in part of the B $\beta 7$ strand. This Asp126-Arg190 salt bridge is located precisely at the entrance of the active site where the CCA-end of tRNA will approach. It is not unreasonable to assume that the CCA-end of the incoming tRNA would stimulate the movement of Arg190, resulting in disruption of the salt bridge, relaxing the distortion of strand $B \beta 7$, and finally opening the glutamic gate. This scenario might be a shared feature between GatCAB and GatDE, because these relevant residues are definitely conserved in both molecules.

Our structural data, however, remains the nature and specificity of binding the tRNA substrate unsolved. We constructed a series of the in vitro transcribed S. aureus (Sav) tRNA $^{\text {Gln }}$ mutants based on the differences between tRNA ${ }^{\text {Gln }}$ and tRNA ${ }^{\text {Glu }}$, and investigated the tRNA-binding activity with the gel-shift assay (Fig. 3, A and B). GatCAB bound quite specifically to tRNA ${ }^{\text {Gln }}$ but not tRNA ${ }^{\text {Glu }}$. Unexpectedly, unlike GluRS or GlnRS, GatCAB did not bind nor recognize the anticodon of tRNA; whereas swapping the upper part of the acceptor stem of tRNA ${ }^{\text {Gln }}$ to tRNA ${ }^{\text {Glu }}$ diminished the binding activity to GatCAB. Changing only the first U1-A72 base pair of tRNA ${ }^{\text {Gln }}$ almost extinguished the binding capacity. This is consistent with the fact that the first U1-A72 base pair is absolutely conserved and unique to tRNA ${ }^{\text {Gln }}$ and tRNA ${ }^{\text {Asn }}$ in bacteria (27) (fig. S5). No binding activity could be detected in the case of the D-arm swapped mutant. The differences in the D-arms between tRNA ${ }^{\text {Gln }}$ and tRNA ${ }^{\text {Glu }}$ are the size of the D-loop and the base pair in the middle of the D-stem (Fig. 3A). Further mutational analysis of the D-arm revealed that the U-insertion in the D-loop was lethal to tRNA recognition. These results suggested that the morphology of the D-loop is important for discrimination between tRNA ${ }^{\text {Gln }}$ and tRNA ${ }^{\text {Glu }}$ by GatCAB. Taken together, GatCAB recognizes and discriminates two identity elements on tRNA ${ }^{\text {Gln }}$; the U1-A72 base pair is exploited as the positive determinant to discriminate tRNA $^{\text {Gln }}$ from the other tRNA species, whereas the U-insertion in the D-loop serves as the negative determinant for distinguishing tRNA ${ }^{\text {Glu }}$ from tRNA ${ }^{\text {Gln }}$. 
It is unclear how GatCAB discriminates the $\mathrm{U} 1-\mathrm{A} 72$ base pair in $\mathrm{tRNA}^{\mathrm{Gln}}$ from the G-C base pair. Interestingly, in the crystal structure of Escherichia coli GlnRS-tRNA ${ }^{\text {Gln }}$ complex, the first U1-A72 base pair of tRNA ${ }^{\text {Gln }}$ was disrupted by the Leu136 residue at the tip of a $\beta$-turn in the acceptor-binding domain of GlnRS (28). GatCAB may use a similar mechanism to distinguish A-U from G-C base pairs by the smaller free energy cost of base-pair denaturation. If this is the case with GatCAB, two turn loops one between $\mathrm{B} \beta 11$ and $\mathrm{B} \alpha 2$ and the other between $\mathrm{B} \beta 12$ and $\mathrm{B} \alpha 3$, are good presumable candidates. On the other hand, we found that the C-terminal helical domain (CHD) of GatB has a crucial role in binding and discrimination of tRNA ${ }^{\mathrm{Gln}}$ (Fig. 3C). The mutant with deletion of the entire domain completely failed to bind to tRNA ${ }^{\text {Gln }}$. Notably, deletion of the conserved Leu472 residue, the fourth residue from the $\mathrm{C}$-terminus, of GatB also caused loss of tRNA-binding activity, whereas a GatB deletion mutant harboring Leu472 did not impair binding activity. Because dozens of hydrophobic residues responsible for inter-helical interactions are highly conserved in CHD, we concluded that elimination of Leu472 disrupted the intact configuration of the last three helices (which were not visible in the electron density map) of CHD. This idea is corroborated by the unusually slow migration of Leu472-deleted mutant protein in our gel-shift experiment. This distal three-helix bundle is connected to the $\mathrm{B} \alpha 11$ helix by a long flexible linker partially seen in the present structure and expected to be able to reach and bind to the D-loop of bound tRNA ${ }^{\mathrm{Gln}}$. Discrimination of the size or configuration of the D-loop of tRNA ${ }^{\text {Gln }}$ may be achieved by fitting to the concave made by helical bundle as a mean of an "indirect readout" mechanism (29).

On the basis of our crystal structures combined with the evident biochemical data, we can extract a simple rationale for the operation of GatCAB controlling the Glu-tRNA $^{\text {Gln }}$-dependent amidotransferase process (fig. S6). Glu-tRNA ${ }^{\text {Gln }}$ is successfully accommodated by GatB through two checkpoints on the body of tRNA ${ }^{\text {Gln }}$; the U1-A72 base pair and the D-loop. Subsequent precise coordination of the carboxylic acid moiety of 
glutamate, attached to the terminal adenosine of tRNA, to primary $\mathrm{Mg}^{2+}$ could induce local conformational remodeling of the active site, shifting the Glu125 gate to open the ammonia channel. This motion may slightly accelerate the glutaminase activity of GatA because of the occurrent flow of ammonia. However, the full activation depends strictly on the recruitment of ATP from the opposite site of the active center. Replacement of the wat95 molecule with the secondary $\mathrm{Mg} 2 \mathrm{t}$ makes the active center ready for the transamidase reaction by preventing g-phosphoryl-glutamate from hydrolysis. Consumption of the ammonia by the transamidase reaction would robustly drive the total potential activity of GatCAB by pumping ammonia downward with a concentration gradient. Our proposed model provides a detailed hypothesis to explain the previous biochemical and kinetic studies of the coupling reaction cycle of $\operatorname{GatCAB}(11,12)$; however, further biochemical and structural works on GatCAB/Glu-tRNA ${ }^{\text {Gln }}$ complex may be needed to complete the scenario of the reaction scheme of adding glutamine to the genetic code. 


\section{References and Notes}

1. $\quad$ M. Ibba, D. Söll, Science 286, 1893 (1999).

2. M. Wilcox, M. Nirenberg, Proc. Natl. Acad. Sci. U. S. A. 61, 229 (1968).

3. M. Wilcox, Eur. J. Biochem. 11, 405 (1969).

4. A. Schon, H. Hottinger, D. Söll, Biochimie 70, 391 (1988).

5. M. Ibba, H. D. Becker, C. Stathopoulos, D. L. Tumbula, D. Söll, Trends. Biochem. Sci. 25, $311(2000)$.

6. $\quad$ A. W. Curnow, M. Ibba, D. Söll, Nature 382, 589 (1996).

7. H. D. Becker et al., FEBS Lett. 476, 140 (2000).

8. $\quad$ A. W. Curnow et al., Proc. Natl. Acad. Sci. U. S. A. 94, 11819 (1997).

9. D. L. Tumbula, H. D. Becker, W. Z. Chang, D. Söll, Nature 407, 106 (2000).

10. E. Schmitt, M. Panvert, S. Blanquet, Y. Mechulam, Structure (Camb) 13, 1421 (2005).

11. L. Feng, K. Sheppard, D. Tumbula-Hansen, D. Söll, J. Biol. Chem. 280, 8150 (2005).

12. K. Y. Horiuchi et al., Biochemistry 40, 6450 (2001).

13. M. Yao, Y. Zhou, I. Tanaka, Acta Crystallogr D. Biol. Crystallogr. 62, 189 (2006).

14. A. T. Brunger et al., Acta Crystallogr. D 54, 905 (1998).

15. Materials and methods are available as supporting material on Science Online.

16. S. Shin et al., EMBO J. 21, 2509 (2002).

17. D. Jahn, Y. C. Kim, Y. Ishino, M. W. Chen, D. Söll, J. Biol. Chem. 265, 8059 (1990).

18. F. C. Wedler, P. D. Boyer, J. Biol. Chem. 247, 984 (1972).

19. J. B. Hunt, A. Ginsburg, Biochemistry 11, 3723 (1972).

20. E. R. Stadtman et al., Mol. Biol. Biochem. Biophys. 32, 144 (1980).

21. D. Eisenberg, H. S. Gill, G. M. Pfluegl, S. H. Rotstein, Biochim. Biophys. Acta 1477, 122 (2000).

22. T. M. Larsen et al., Biochemistry 38, 16146 (1999).

23. A. Douangamath et al., Structure 10, 185 (2002).

24. A. K. Bera, J. L. Smith, H. Zalkin, J. Biol. Chem. 275, 7975 (2000).

25. S. Khademi et al., Science 305, 1587 (2004).

26. O. M. Petrek, P. Banas, J. Koca, J. Damborsky, CAVER program, http://loschmidt.chemi.muni.cz/caver/index.php.

27. C. Marck, H. Grosjean, RNA 8, 1189 (2002).

28. M. A. Rould, J. J. Perona, D. Söll, T. A. Steitz, Science 246, 1135 (1989).

29. S. Hauenstein, C. M. Zhang, Y. M. Hou, J. J. Perona, Nat. Struct. Mol. Biol. 11, 1134 (2004).

30. We thank Y. Tanaka for helpful advice in initial molecular cloning and purification processes of GatCAB complex, and the staff of beamlines BL41XU and BL44B2 at SPring-8, as well as of ARNW12 station at Proton Factory, for help during data collection. We are grateful to O. Nureki (Tokyo Institute of Technology) for providing the refined coordinates of GatDE/tRNA ${ }^{\text {Gln }}$ complex before publication. This work was supported by the National Project on Protein Structural and Functional Analyses from the Ministry of Education, Culture, Sports, Sciences and Technology of Japan, and was supported in part by a Human Frontiers Science Program Research grant. Coordinates and structure-factor amplitudes have been deposited in the Protein Data Bank under code 2G5H, 2F2A, 2G5I, 2DF4 and 2DQN for the apo form, and the glutamine-bound, ATP analog-bound, $\mathrm{Mn}^{2+}$-bound, and asparagines-bound forms, respectively. 


\section{Figure legends}

Fig. 1. Bacterial GatCAB complex fastens a molecular belt. (A) Front view ribbon diagram of the overall structure of $S$. aureus GatCAB/glutamine complex at $2.3 \AA$ resolution, depicted in three different colors for each subunit; blue, green, and magenta for GatA, GatB, and GatC, respectively. Glutamine in the active site of GatA is drawn as yellow stick representations, whereas the purple sphere is the magnesium ion found in the active site of GatB. ADP from the cocrystal structure with $\mathrm{ADP}-\mathrm{AlF}_{4}{ }^{-}$is shown together as pink sticks. This color code is used throughout all the figures. (B) Top view of annularly shaped GatC. (C) Amphipathic helices at the N-terminus of GatC form a helical bundle with the hydrophobic core of GatA (hydrophobic residues are colored gray.). (D) Detailed interactions between the internal loop region of GatC and GatAB complex. Conserved residues involved are labeled, with hydrogen-bonds indicated ( $\leq 3.2 \AA$, dashed black line). Polar interactions are prominent on the $\mathrm{C}$-terminal side, whereas the hydrophobic interactions are clustered on the opposite side. (E) The C-terminus of GatC tightens GatAB complex, constructing an antiparallel $\beta$-sheet and a hydrophobic platform.

Fig. 2. A $30 \AA$ long ammonia channel connects the two remote active centers of GatCAB. (A) The active site of a glutaminase reaction in GatA is composed of the conserved Ser-cis-Ser-Lys catalytic scissors shown as magenta stick representations. Residues involved in the hydrogen-bonded network (dashed black lines) in the active site are labeled. A plausible hydrolytic water molecule is colored light blue and is on the opposite side of a supposed ammonia product (orange sphere). The Fo-Fc electron density map (contoured at $3 \sigma$, green mesh) calculated without the glutamine and Ser178 clearly demonstrates the tetrahedral covalent intermediate of the glutamine with Ser178. (B) The environment of the ADP-binding site shown together with the omit $F$ o-Fc electron density map ( $2 \sigma$, blue). Residues contributing to ADP (ball-and-stick) recognition are represented as stick models with labels. Two water molecules (light blue spheres) are coordinated to a magnesium ion (purple) and to $\beta$-phosphate. (C) The putative ammonia channel was calculated using the program CAVER (26), with the structure of the water-omitted GatCAB/glutamine complex. Glu125в blocking the ammonia transport route is shown in a space-filling representation for clarity. The channel was filled with a row of solvent molecules (light blue spheres), which interact with the conserved polar residues (colored sticks) along the pathway. A bound glutamine in GatA is drawn as spheres indicating the start point of the channel. (D) Schematic representation of the ammonia channel. Residues defining the channel are colored corresponding to their properties: red, negative; blue, positive; black, non-polar side chain; gray, main chain. Hydrolyzed ammonia is colored orange. Strictly conserved 
residues are underlined and hydrogen-bonding distances are indicated $(\AA)$. The presumed movement of the Glu125B gate to open the ammonia channel is indicated by a black arrow.

Fig. 3. Mutational analyses of tRNA ${ }^{\mathrm{Gln}}$ and GatCAB. (A) Cloverleaf representations of tRNA $^{\text {Gln }}$ (black) and tRNA ${ }^{\text {Glu }}$ (gray) from S. aureus. Indicated bases or stem-loops (magenta) were altered to those of tRNA ${ }^{\text {Glu }}$ to verify the binding activity. Bases involved in specific recognition by GatCAB are emphasized and marked with blue stars. (B) Gel-shift assay with tRNA ${ }^{\text {Gln }}$ variants as specified in (A). Bands corresponding to $150 \mathrm{pmol}^{\mathrm{tRNA}}{ }^{\mathrm{Gln}}$ and 50 pmol GatCAB are shown in the first and second lanes, respectively. The molecular ratios of the enzyme to tRNA ${ }^{\text {Gln }}$ were fixed at 1:3. Uncharacterized bands above tRNA were probably responsible for the tRNA dimer suggested by the results of size exclusion chromatography in our experiments. Gels were stained with Coomassie brilliant blue and sequentially with tluidine blue to visualize proteins and RNA, respectively. (C) A series of GatB deletion mutants (50 pmol GatCAB complex per lane) were subjected to gel-shift assay in the absence $(-)$ or presence $(+)$ of 150 pmol of the wild-type tRNA ${ }^{\text {Gln }}$. Only Leu472 harboring $\Delta 3$ mutant (deletion of three residues from C-terminus of GatB) retained the same binding activity as wild-type (WT) GatCAB. Deletion of Leu472 from the C-terminus of GatB ( $\Delta 4$ mutants) abolished the tRNA-binding capacity. Further deleted mutant $(\Delta 10)$ as well as a mutant lacking the helical bundle domain $(\triangle \mathrm{CHD})$ of GatB did not show any tRNA-binding activity. 
A

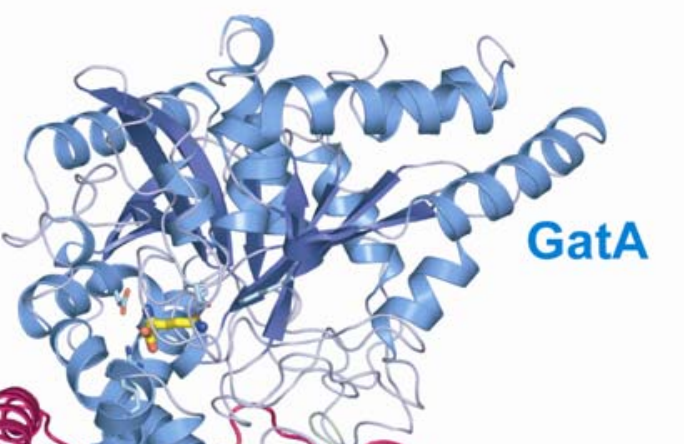

B
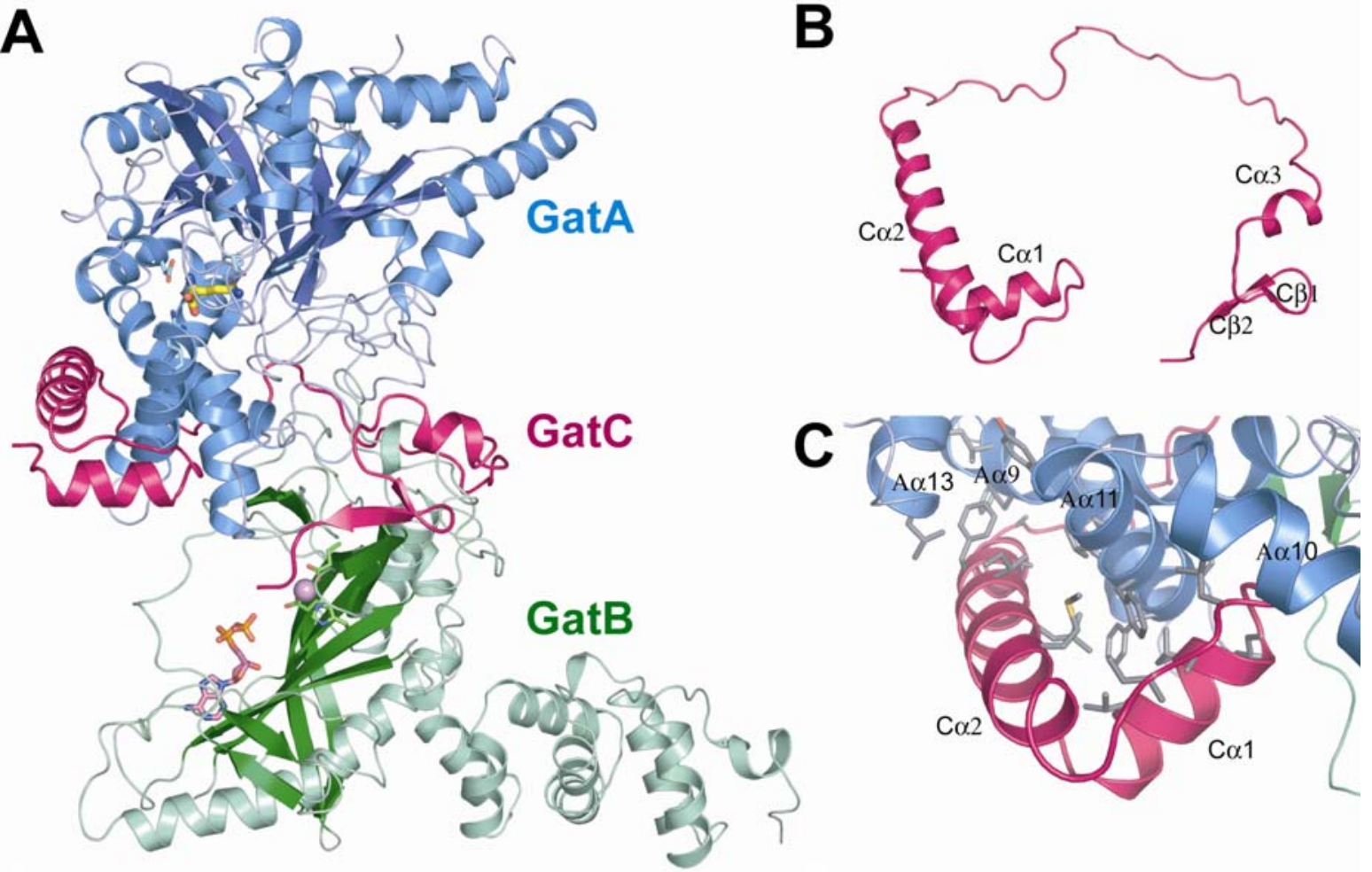

D
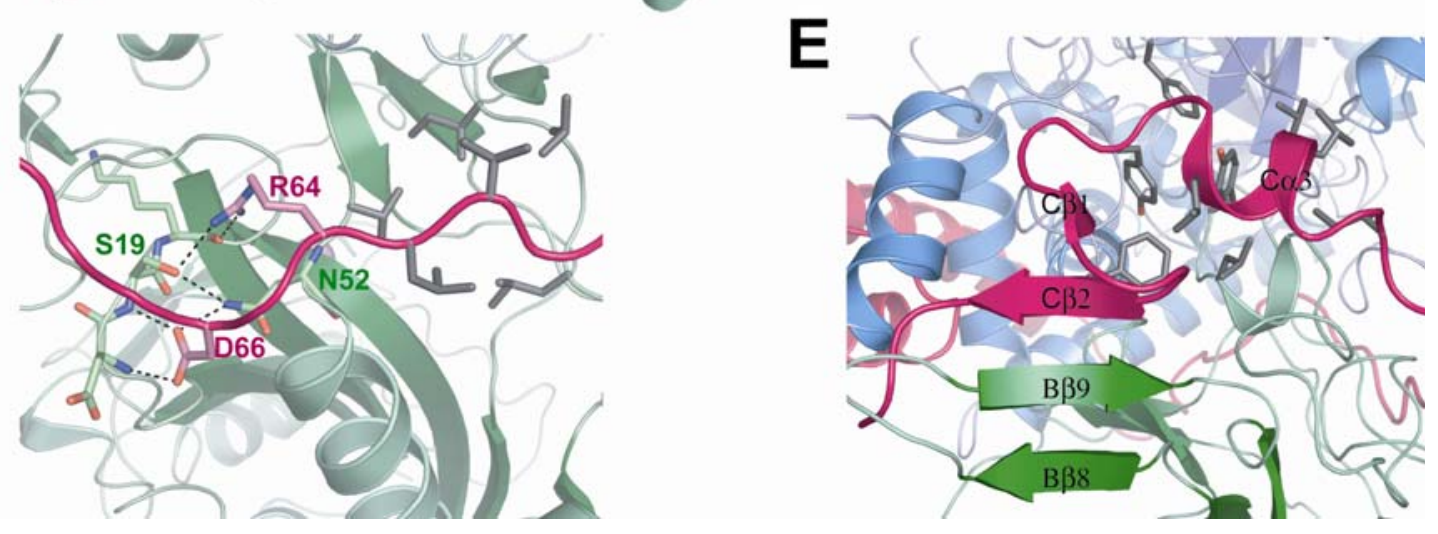

Fig. 1. 


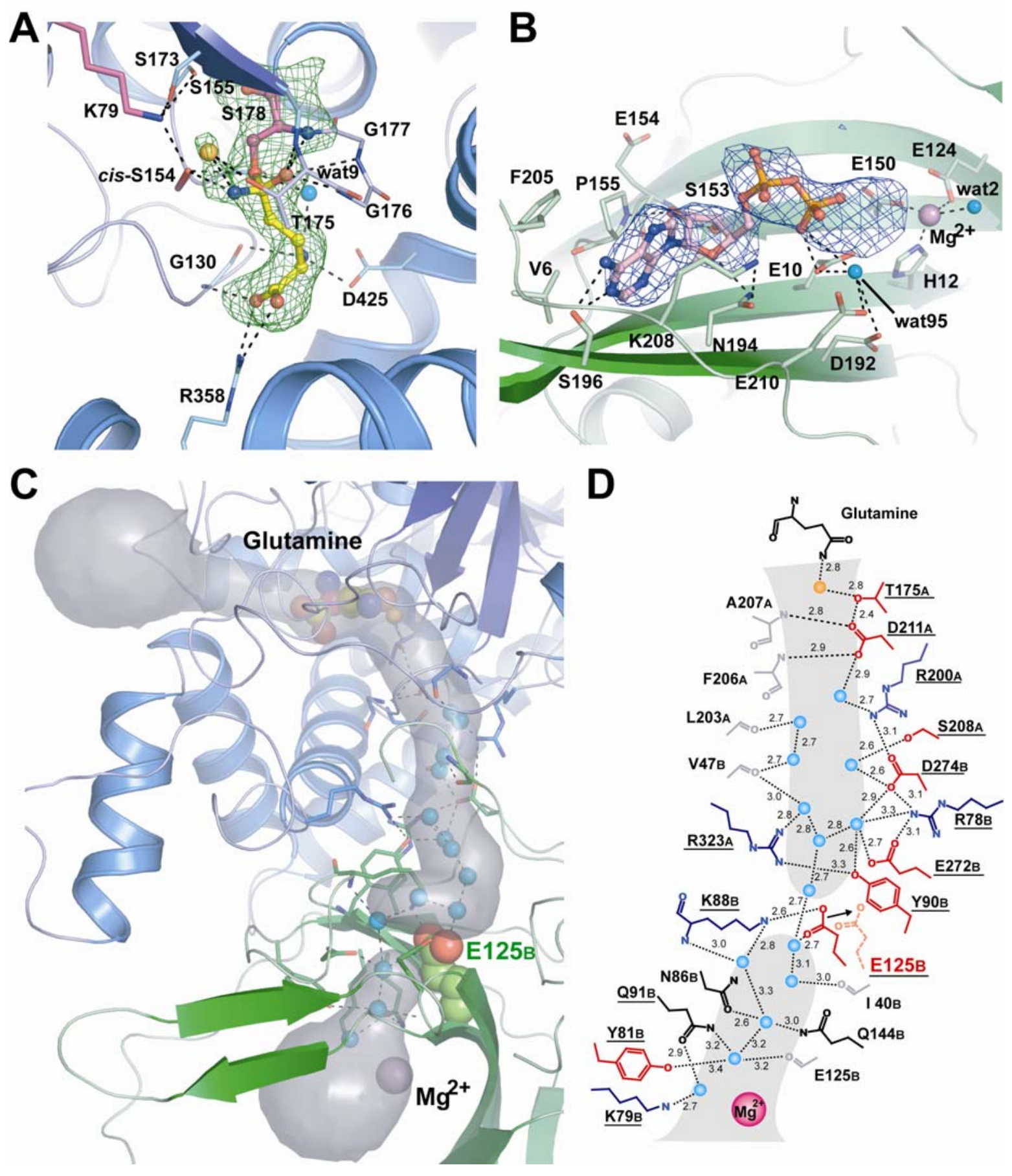

Fig. 2. 
A

B
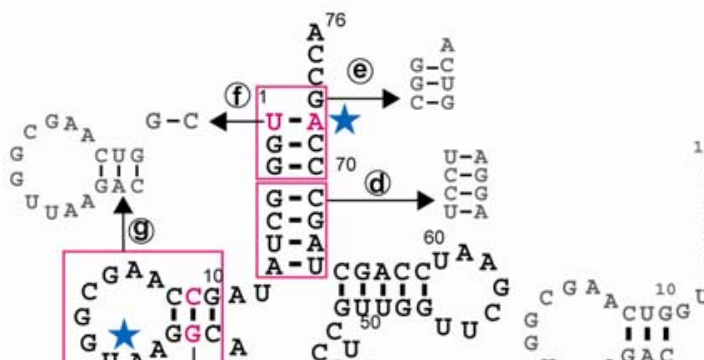

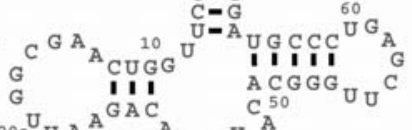

$\mathrm{C}_{\mathrm{gRNa}}$

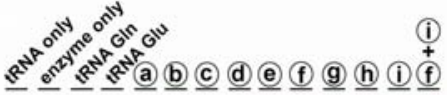

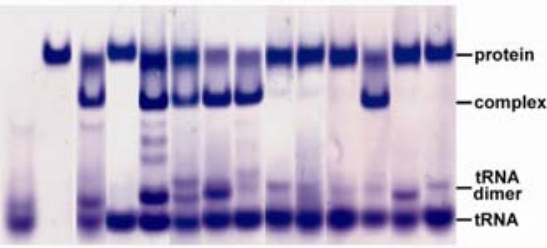

(i)

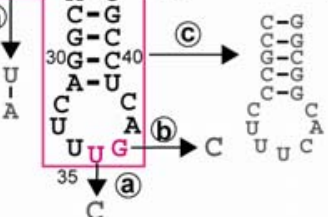

${ }^{C}=G^{-}$

$\mathrm{G}=$

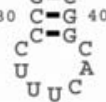

S. aureus tRNA ${ }^{\mathrm{GIn}}$

S. aureus tRNA Glu

Fig. 3. 